\title{
Avascular necrosis of the hip in a man with HIV infection
}

\author{
B P Goorney, H Lacey, S Thurairajasingam, J D K Brown
}

\begin{abstract}
The development of an osteonecrotic (avascular necrosis) hip joint in a patient with symptomatic CDC Stage $1 \mathrm{~V}$ C2 disease is reported. The diagnosis was aided by radiological assessment and confirmed by radioisotope bone scan. This case serves as yet another musculoskeletal manifestation in association with HIV infection.
\end{abstract}

The Departments of Genitourinary Medicine ${ }^{\star}$ and Radiology, Hope Hospital, University of Manchester School of Medicine, Salford M6 8HD, UK

B P Goorney, ${ }^{*}$ L Lacey, ${ }^{\star} S$ Thurairajasingam, J D K Brown

\section{Case report}

A 34 year old man known to be HIV-antibody positive first presented to the genitourinary medicine clinic in Salford, in September 1988. At the time he was found to have oropharyngeal candidiasis with angular cheilitis and a follicular eruption on chest and upper arms. His CD4 T lymphocyte count was $300 / \mathrm{mm}^{3}$ and HIV p24 antigen present in the serum at a level of $120 \mu / \mathrm{ml}$.

The patient was initially commenced on oral fluconazole $50 \mathrm{mg}$ a day and a topical anti-fungal agent for the folliculitis. Subsequent follow up was unremarkable, and he remained free of opportunistic infections. However in December 1989 he complained of vague pain in the right hip, physical examination revealed no abnormality and a

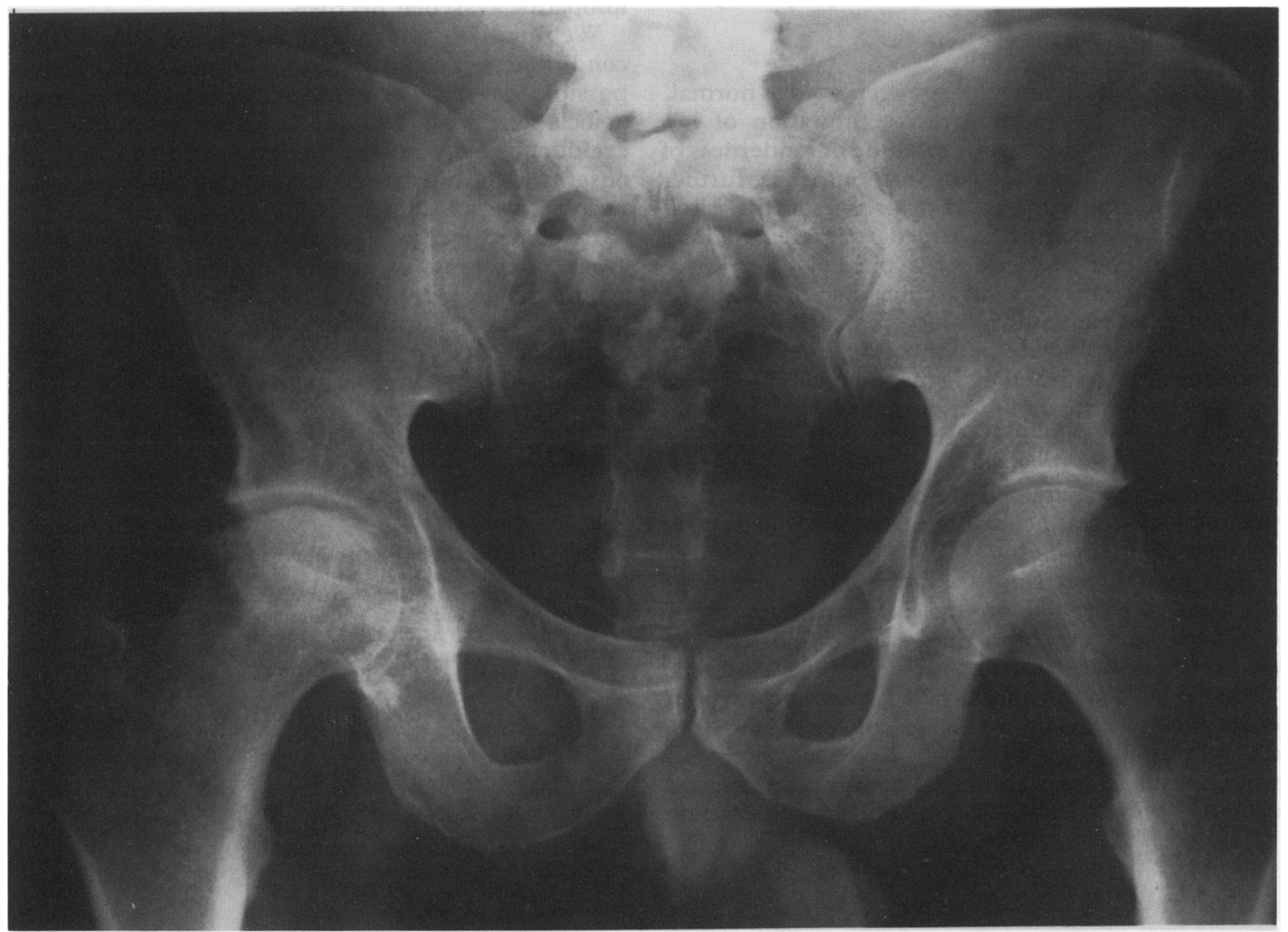

Figure 1 Hip radiograph with osteonecrosis of right femoral head. 


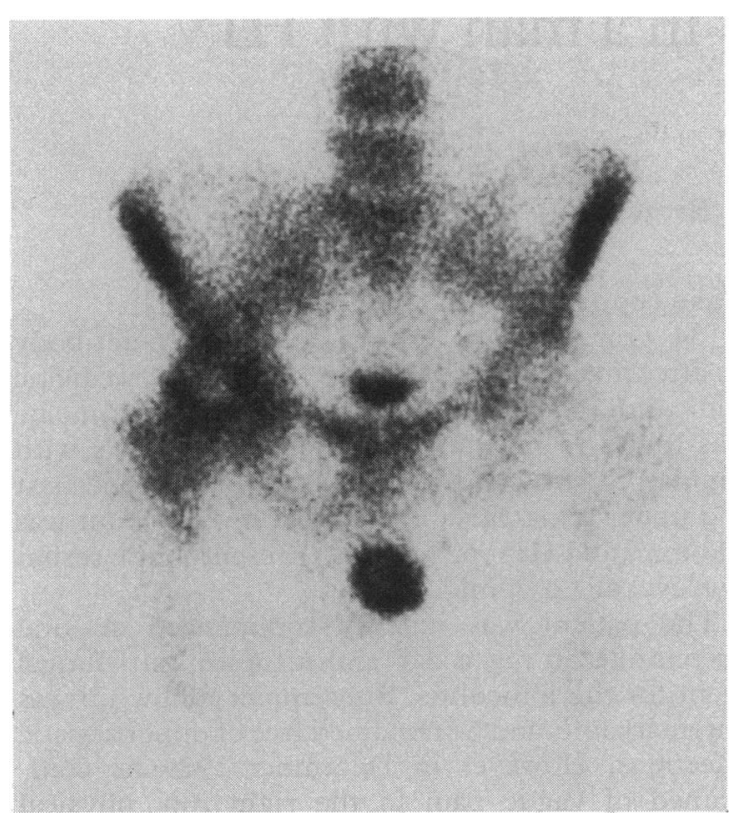

Figure 2 Technecium bone scan showing photon deficient area surrounded by markedly increased isotope uptake of right hip.

radiograph of both hip joints was reported as normal.

There followed a progressive limitation of hip movement, but without any swelling or tenderness of the joint over the following 6 months. A further radiograph showed (fig 1) sclerosis of the right femoral head, with interspersed lytic areas. These findings in the presence of a normal joint space indicate osteonecrosis (avascular necrosis) of the femoral head rather than a septic process. A technecium diphosphonate radionuclide bone scan, was performed to confirm the diagnosis and also to assess the extent of the bone disease (fig 2). The scan revealed a photon deficient area of the femoral head (consistent with necrotic bone but not infection) surrounded by increased isotope uptake, thus confirming avascular necrosis. All other joints were normal.

The patient was admitted for bed rest and analgesia and investigated for a possible underlying cause of his osteonecrotic hip. Apart from a transient low grade fever there were no new physical findings. Tests revealed at the time a CD4 T lymphocyte count of $200 / \mathrm{mm}^{3}$, haemoglobin $15.4 \mathrm{~g} / \mathrm{l}$, platelets $94 \times 10^{9} / 1$, and white cell count $4.6 \times 10^{9} / 1$. Liver function tests were normal as were serum calcium, phosphate and alkaline phosphatase. Blood cultures including culture for mycobacteria were negative. There was no previous history of trauma, or hip disease in childhood.

After several weeks of treatment there was significant improvement in hip movement, and he was able to bear weight. He was also commenced on zidovudine $1 \mathrm{~g}$ a day and at follow up the patient remains well, is apyrexial and has improved mobility.

\section{Discussion}

Rheumatic manifestations of HIV disease are welldocumented $^{12}$ all of which may involve the hip joint. ${ }^{3}$ To our knowledge there is no previously reported association between avascular necrosis of the hip and symptomatic HIV infection.

Avascular necrosis of the hip is commonly associated with a number of underlying conditions such as Gaucher's disease and Perthe's disease, the pathogenesis of which is that of fat embolism. ${ }^{4}$ Other conditions are haemoglobinopathies, hyperbarism, hypercortisonism and major trauma resulting in vascular occlusion of intracapsular arteries. ${ }^{45}$ In a minority of cases no cause is found, or it is felt related to alcohol abuse or obesity, and is known as idiopathic avascular necrosis. ${ }^{5}$

We found no evidence of any of these underlying conditions, as a cause of his hip disease. Although it is possible that this was an incidental finding, a genuine association with his HIV infection cannot be excluded. The symptoms were vague and insidious in onset, and the hip disease was not detected until bone necrosis was advanced.

In conclusion we feel that clinicians should be aware of unusual or atypical presentations of joint symptoms in symptomatic HIV individuals, particularly involving the hip joint and that a careful radiological assessment and early diagnosis may limit the subsequent damage to the joint as a result of osteonecrosis.

\section{Address correspondence to Dr BP Goorney.}

1 Rowe IF, Keat AC. Human immunodeficiency virus infection and the rheumatologist. Ann Rheum Dis 1989;48:89-91.

2 Rowe IF, Forster SM, Seifert $M H$, et al. Rheumatological lesions in individuals with human immunodeficiency virus infection. $Q J$ Med 1989;73:1167-84.

3 Berman A, Espinoza LR, Diaz JD, et al. Rheumatic manifestations of human immunodeficiency virus. $A m J M e d$ 1988;85:59-64.

4 Jones JP Jr. Fat embolism and osteonecrosis. Orthop Clinics North Am 1985;16:595-633.

5 Soloman L. Mechanisms of idiopathic Osteonecrosis. Orthop Clin North Am 1985;16:655-67.

Accepted for publication 15 August 1990 